14

\title{
Адаптивная фильтрация сигналов электроэнцефалограмм с использованием метода эмпирических мод
}

\author{
(ㄱ В.В. Грубов, А.Е. Руннова, А.А. Короновский, А.Е. Храмов
}

Саратовский государственный технический университет им. Гагарина Ю.А.

Саратовский государственный университет им. Н.Г. Чернышевского E-mail: vvgrubov@gmail.com, hramovae@gmail.com

Поступило в Редакцию 9 марта 2017 г.

Разработан новый метод для удаления физиологических артефактов на экспериментальных сигналах электроэнцефалограмм (ЭЭГ) человека. Метод основан на процедуре разложения сигнала по эмпирическим модам. Алгоритм метода включает разложение сигнала ЭЭГ по эмпирическим модам, поиск мод с артефактами, удаление этих мод и восстановление сигнала ЭЭГ. Метод был протестирован на экспериментальных данных и показал высокую эффективность при удалении различных физиологических артефактов на ЭЭГ.

DOI: 10.21883/PJTF.2017.13.44812.16766

В настоящее время наблюдается значительный интерес к изучению колебательной активности в нейронных сетях головного мозга. Основными источниками информации о работе мозга являются экспериментальные методы регистрации активности головного мозга, например электроэнцефалограммы (ЭЭГ) [1]. Сигнал ЭЭГ имеет сложный состав с рядом характерных ритмов и паттернов, которые представляют интерес для исследователей как при изучении патологий (например, эпилепсии), так и при анализе когнитивных процессов $[2,3]$.

В радиофизике разработан ряд методов анализа нестационарных сигналов, например, оконное фурье-преобразование или непрерывный вейвлет-анализ [4], которые оказываются весьма эффективными при анализе ЭЭГ $[5,6]$. Тем не менее в большинстве случаев исследование сигналов ЭЭГ осложняется наличием паразитных паттернов - шумов и 
артефактов, которые вызваны как внешними источниками сигналов, так и процессами, протекающими в самом организме, например, движением глаз, кардиоритмами, активностью лицевых и шейных мышц и т. д. [7,8].

Большинство артефактов на ЭЭГ имеют значительную амплитуду и перекрывают три важных низкочастотных диапазона ЭЭГ - дельта, тета и альфа $[1,2]$. Присутствие артефактов и их вариабельность значительно усложняют анализ сигналов ЭЭГ, что делает предварительную обработку и фильтрацию важным этапом любых исследований ЭЭГ.

Для фильтрации ЭЭГ от артефактов применяют ряд различных методов: на базе визуального поиска артефактов $[9,10]$, анализа независимых компонент $[3,11,12]$, регрессионного анализа [13], преобразования Грамма-Шмидта [14]. Большинство методов вызывают искажения сигнала ЭЭГ [15] или требуют совместного анализа ЭЭГ с другими сигналами, которые не всегда могут быть записаны в ходе эксперимента.

Важной задачей является разработка методов фильтрации сигналов ЭЭГ, не искажающих их структуру и не требующих записи дополнительных физиологических сигналов. В настоящей работе предложен новый метод удаления артефактов на ЭЭГ, основанный на разложении по эмпирическим модам (ЭМ) [16,17].

Разложение сигнала по ЭМ является одним из современных методов частотно-временного анализа нелинейных нестационарных сигналов и позволяет представить исследуемый сигнал в виде набора амплитудномодулированных компонент с нулевым средним - так называемых эмпирических мод. В разложении по ЭМ базисные функции определяются из самого сигнала, а их характеристики также напрямую зависят от исследуемого сигнала. Это свойство делает разложение по ЭМ высокоадаптивным инструментом анализа сигналов. Исследования показывают [18], что во многих случаях частотно-временной анализ и выделение конкретных осцилляторных паттернов (включая артефакты) могут быть сведены к анализу одной или нескольких ЭМ сигнала ЭЭГ.

Данное свойство проиллюстрировано на рис. 1, на котором приведен экспериментальный сигнал ЭЭГ человека $(a)$ с несколькими глазодвигательными артефактами, а также первые три ЭМ для него $(b, c, d)$. Дополнительно на рис. 1 представлены вейвлет-спектры, построенные с Морле-вейвлетом, которые используются для представления частотновременной структуры сигналов. Из вейвлет-поверхности на рис. 1,a видно, что исходный сигнал ЭЭГ содержит различные ритмы в диапазоне 0.5-50 Hz, в то время как артефакты возникают в диапазоне

Письма в ЖТФ, 2017, том 43, вып. 13 


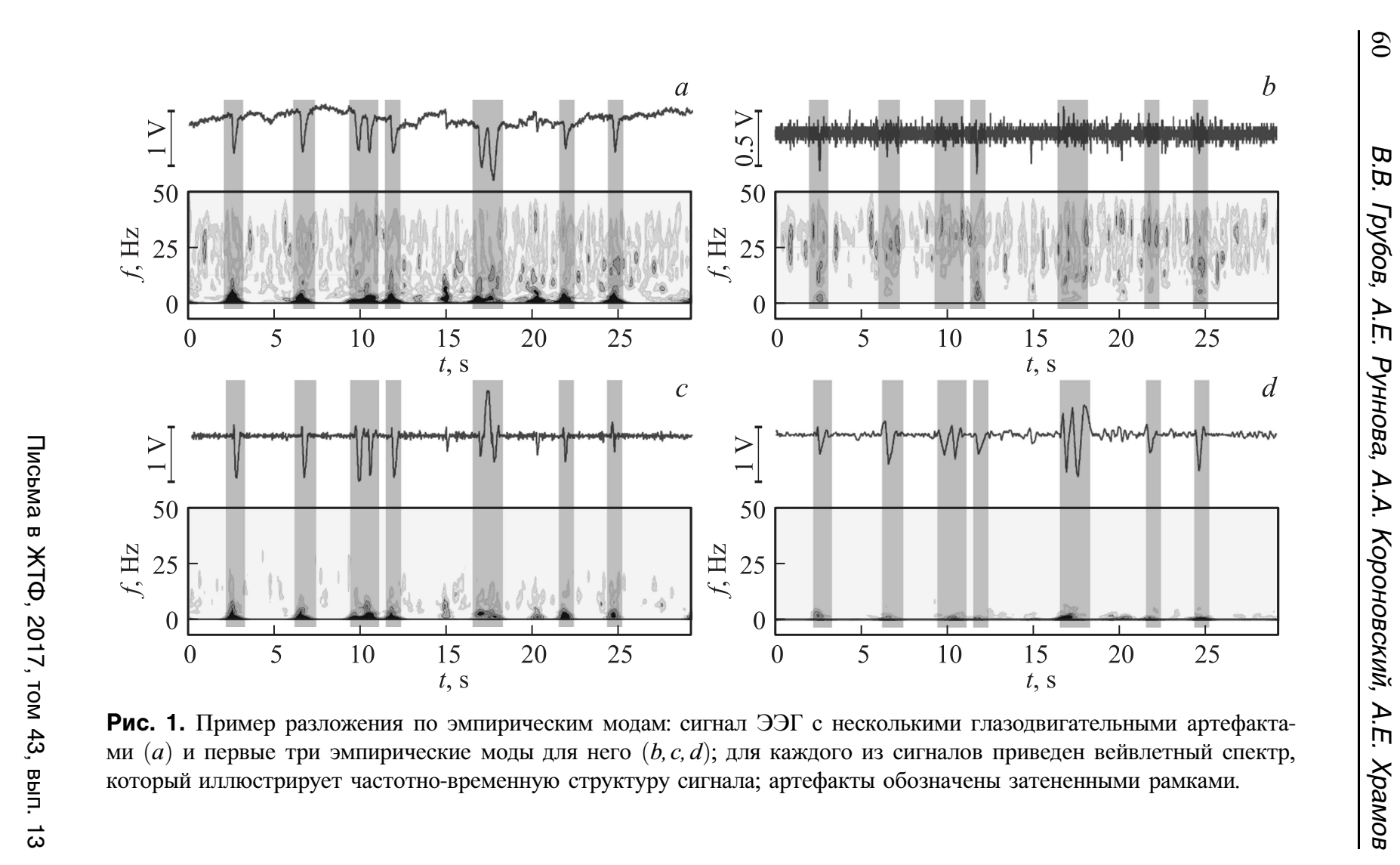


0.5-5 Hz. Вейвлет-спектр первой ЭМ (рис. $1, b)$ демонстрирует самые высокие частоты, которые соответствуют информативным компонентам сигнала ЭЭГ. Рис. $1, c, d$ содержит вторую и третью ЭМ сигнала ЭЭГ вместе с их вейвлетными спектрами, которые в основном состоят из низких $(\sim 0.5-5 \mathrm{~Hz})$ частот и соответствуют фоновой ЭЭГ активности и глазодвигательным артефактам. Таким образом, в данном случае глазодвигательные артефакты могут быть локализованы во второй и третьей ЭМ, в то время как первая ЭМ соответствует сигналу ЭЭГ, очищенному от артефактов. Данная процедура локализации артефактов на ЭЭГ была использована в качестве ключевого элемента при разработке нового метода фильтрации сигналов ЭЭГ.

Алгоритм предложенного метода имеет следующий вид:

1. Разложение исследуемого сигнала ЭЭГ на набор ЭМ.

2. Нахождение ЭМ, содержащих физиологические артефакты.

3. Удаление ЭМ, содержащих физиологические артефакты.

4. Восстановление сигнала ЭЭГ из оставшихся ЭМ.

На первом этапе алгоритма производится разложение сигнала ЭЭГ по ЭМ, а также определяется общее количество ЭМ. На втором этапе алгоритма среди рассматриваемых ЭМ производится поиск мод, содержащих артефакты. Для этого сравниваются вейвлет-спектры исходного сигнала ЭЭГ и ЭМ. Из исследований ЭЭГ известно, что большая часть физиологических артефактов обладает специфическими частотновременными характеристиками, которые в совокупности создают характерный образ на вейвлет-спектре для каждого типа артефакта. В предложенном методе образы артефактов сначала определяются на вейвлетном спектре исходного сигнала ЭЭГ, а затем анализируются вейвлетные спектры каждой отдельной ЭМ. На третьем этапе алгоритма все ЭМ, на вейвлет-спектрах которых были обнаружены образы артефактов, удаляются из рассмотрения. На четвертом этапе производится восстановление сигнала ЭЭГ путем суммирования оставшихся ЭМ. Результатом работы предложенного метода является восстановленный сигнал ЭЭГ, на котором удалены физиологические артефакты.

Разработанный метод был протестирован на примере удаления физиологических артефактов двух типов с экспериментальных сигналов ЭЭГ человека. Сигналы ЭЭГ были записаны при помощи стандартной схемы расстановки электродов - международной системы 


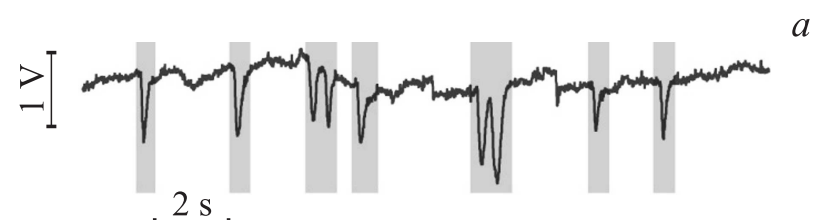

$2 \mathrm{~s}$

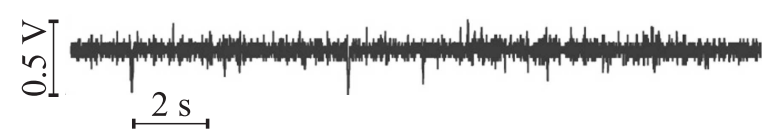

$b$

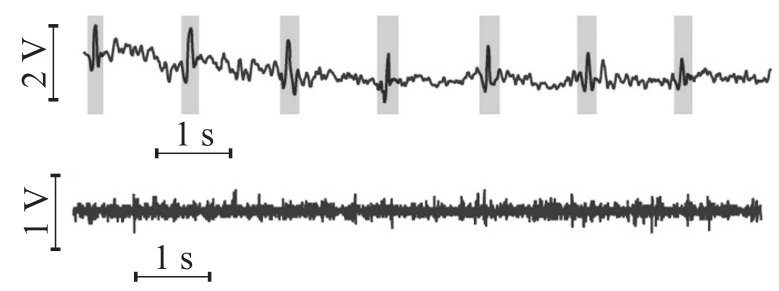

Рис. 2. Пример фильтрации сигнала ЭЭГ от артефактов двух типов: глазодвигательных $(a)$ и кардиоритмов $(b)$; сигналы ЭЭГ до фильтрации приведены в левой части рисунка, сигналы после фильтрации - в правой; артефакты обозначены затененными рамками.

„10-20“ [19]. Все эксперименты включали в себя стандартные физиологические пробы и были проведены для 15 здоровых мужчин и женщин в возрасте $18-40$ лет.

В ходе проведения экспериментов на записях ЭЭГ было обнаружено два типа артефактов: глазодвигательные и кардиоритмы. Оба типа артефактов имеют сходную форму и проявляются в виде коротких высокоамплитудных всплесков активности.

Пример работы предложенного метода представлен на рис. 2, на котором приведены экспериментальные сигналы ЭЭГ человека, содержащие глазодвигательные артефакты $(a)$ и кардиоритмы $(b)$. Также на рис. 2 представлены сигналы ЭЭГ после фильтрации. Видно, что в каждом из случаев были удалены как артефакты, так и низкочастотная огибающая сигнала ЭЭГ, которая не содержит полезной информации. Таким образом, предложенный метод может быть использован не только 
для удаления различных типов артефактов на ЭЭГ, но и для фильтрации шумовых компонент.

Эффективность разработанного метода была продемонстрирована на примере удаления глазодвигательных артефактов с экспериментальной записи ЭЭГ человека продолжительностью 600 s c 95 артефактами амплитудой от 1 до $4 \mathrm{~V}$. Критерием удаления артефакта было снижение его амплитуды после фильтрации до уровня средней амплитуды сигнала ЭЭГ (в данном случае $0.6 \mathrm{~V}$ ). В ходе фильтрации с записи ЭЭГ было удалено 88 артефактов, и точность разработанного метода составила $\sim 92 \%$. Кроме того, у артефактов, которые не были удалены полностью, была значительно снижена амплитуда (вплоть до 70\% от исходной амплитуды), что также полезно для фильтрации сигнала ЭЭГ.

При анализе эффективности метода была рассчитана количественная характеристика искажения спектра сигнала до и после фильтрации $M$. Для этого были рассчитаны вейвлетные спектры в диапазоне $\Delta f=5-15 \mathrm{~Hz}$ для исходного и фильтрованного сигналов ЭЭГ и $M$ была рассчитана как

$$
M=\iint_{\Delta f}^{\tau} \int_{0}^{\tau}\left|W\left(f, t_{0}\right)-W_{E M}\left(f, t_{0}\right)\right| d t d f
$$

где $W\left(f, t_{0}\right)$ и $W_{E M}\left(f, t_{0}\right)$ - амплитуды вейвлет-спектра сигнала ЭЭГ до и после фильтрации соответственно, $\tau$ - длина сигнала ЭЭГ. Было получено $M<10^{-2}$, а следовательно, искажения сигнала ЭЭГ в ходе удаления артефактов можно считать незначительными.

Таким образом, в настоящей работе был предложен новый метод для фильтрации и удаления физиологических артефактов с экспериментальных сигналов ЭЭГ. Был разработан алгоритм метода, основанный на использовании разложения сигнала по эмпирическим модам. Метод был апробирован на примере удаления артефактов двух типов на сигналах ЭЭГ и показал высокую эффективность.

Работа выполнена при поддержке РФФИ (грант 16-29-08221 офи_м), грантов президента РФ МК-3305.2017.2 и Министерства образования и науки РФ 3.861.2017/ПЧ.

Письма в ЖТФ, 2017, том 43, вып. 13 


\section{Список литературы}

[1] Nunez P.L., Srinivasan R. Electric fields of the brain: the neurophysics of EEG. Oxford University Press, 2006. 640 p.

[2] Buzsaki G., Draguhn A. // Science. 2004. V. 304. P. 1926-1929.

[3] van Luijtelaar G., Lüttjohann A., Makarov V.V. et al. // J. Neurosci. Methods. 2016. V. 260. P. 144-158.

[4] Hramov A.E., Koronovskii A.A., Makarov V.A. et al. Wavelets in Neuroscience. Heidelberg, New York, Dordrecht, London, 2015. 318 p.

[5] Павлов А.Н., Храмов А.Е., Короновский А.А. et al. // УФН. 2012. Т. 182. № 9. C. 905-939.

[6] Божсокин С.В., Суслова И.М. // ЖТФ. 2013. Т. 83. В. 12. С. 26.

[7] Ebersole J.S., Husain A.M., Nordli D.R. Current Practice of Clinical Electroencephalography. Wolters Kluwer, 2014. 664 p.

[8] Luders H., Noachtar S. Atlas and Classification of Electroencephalography. Philadelphia: WB Saunders Co., 2000. 208 p.

[9] Zhang C., Tong L., Zeng Y. et al. // Biomed Res. Int. 2015. P. 720450.

[10] Uriguen J.A., Garcia-Zapirain B. // J. Neural Eng. 2015. V. 12. N 3. P. 031001.

[11] Bell A.J., Sejnowski T.J. // Neural Comput. 1995. V. 7. N 6. P. 1129.

[12] Joyce C.A., Gorodnitsky I.F., Kutas M. // Psychophysiology. 2004. V. 41. N 2. P. 313-325.

[13] Gratton G. // Instruments \& Computers. 1998. V. 30. P. 44-53.

[14] Короновский А.А., Храмов А.Е., Москаленко О.И., Грубов В.В. / Патент на изобретение RUS N 2560388 от 21.07.2015.

[15] Меринов П.А., Беляев М.Г. // Труды 39-й Междисциплинарной школыконференции ИППИ РАН „Информационные технологии и системы 2015“. М.: Изд-во Института проблем передачи информации им. А.А. Харкевича PAH, 2015. C. 313-328.

[16] Huang N.E., Shen Z., Long S.R. et al. // Proc. R. Soc. A. 1998. V. 454. P. 903.

[17] Павлов А.Н., Филатова А.Е., Храмов А.Е. // Радиотехника и электроника. 2011. T. 56. № 9. C. 1099-1106.

[18] Grubov V.V., Sitnikova E.Yu., Pavlov A.N. et al. // Proc. SPIE. 2015. V. 9448. P. $94481 Q$.

[19] Nuwer M.R., Comi C., Emerson R. et al. // Electroencephalogr. Clin. Neurophysiol. 1998. V. 106. P. 259-261. 